\title{
Reinterpretación del complejo Alstroemeria diluta Ehr. Bayer (Alstroemeriaceae) basada en el color y ornamentación de los tépalos, con cambio de distribución geográfica y de estatus de sus taxones
}

\author{
Reinterpreting the Alstroemeria diluta Ehr. Bayer complex (Alstroemeriaceae) based on color \\ and ornamentation of tepals, with change in geographical distribution and status of its taxa
}

\author{
Mélica Muñoz-Schick ${ }^{1, *} \&$ Andrés Moreira-Muñoz ${ }^{2}$ \\ ${ }^{1}$ Museo Nacional de Historia Natural, interior Quinta Normal, Casilla 787, Santiago, Chile. \\ IInstituto de Geografía, Pontificia Universidad Católica de Valparaíso, Avenida Brasil 2241, Valparaíso, Chile. \\ *melica4@gmail.com
}

\begin{abstract}
The species complex Alstroemeria diluta Ehr. Bayer is evaluated according to a reinterpretation of the color and ornamentation of the tepals. As such, change in the distribution and a new status of infraespecific taxa are proposed.
\end{abstract}

El género Alstroemeria es estrictamente sudamericano y abarca desde Venezuela $\left(3^{\circ} \mathrm{N}\right)$ hasta Tierra del Fuego $\left(53^{\circ}\right.$ S). La mayor cantidad de taxones se encuentra en Chile (49), luego en Brasil (39), Argentina (10) y Perú (7), mientras que Bolivia, Uruguay, Paraguay y Venezuela tienen una sola especie (Muñoz-Schick \& Moreira-Muñoz 2003). En la nueva Guía sobre este género (Finot et al. 2018), se indican 58 taxones aceptados para Chile y recientemente se agrega un nuevo taxón (Muñoz-Schick et al. 2019).

La variabilidad morfológica floral en el género en Chile es muy grande, una misma especie puede presentar flores de colorido y dibujos altamente diversos. Bayer (1987), en su monografía del género, divide $A$. diluta en dos subespecies e indica que $A$. diluta subsp. chrysantha (ejemplar Tipo recolectado en Ruta 5 norte, $\mathrm{Km} \mathrm{435,} \mathrm{cerca} \mathrm{de} \mathrm{Guanaqueros)}$ se diferencia de A. diluta subsp. diluta (ejemplar Tipo de Talca, Ruta 5 sur, Km 229) por las flores amarillo-oro, de tépalos un poco más anchos y con dibujos purpúreo-maculados en los tépalos internos superiores. En las fotografías que incluye hay dos de Fray Jorge (región de Coquimbo), de tépalos externos color rosado-cárneo, internos amarillos, y una de Guanaqueros (región de Coquimbo) con todos los tépalos amarillos. Siguiendo este criterio, Muñoz-Schick \& MoreiraMuñoz (2003) malinterpretaron A. diluta subsp. chrysantha, ya que tomaron en consideración cualquier tono amarillo presente en los ejemplares.

A. diluta es una especie con tépalos angostamente obovados, de bordes aserrados, color blanco a rosado, de 3-5 cm de largo, los internos superiores algo más angostos que los externos, con rayitas atropurpúreas en la mitad superior, dispersas o uniéndose hacia el ápice, con o sin franja amarilla (Muñoz-Schick \& Moreira-Muñoz 2003).

El objetivo de este artículo es aclarar los taxones en base al colorido de los tépalos. Las observaciones deberían efectuarse en las poblaciones, ya que en una misma población puede notarse gran variabilidad (por ejemplo, en Mantos de Hornillo, región de Coquimbo).

\section{ANTECEDENTES}

Watson y Flores (en Hoffmann, Watson \& Flores 2015) habían planteado la variabilidad de la especie, pero ellos separaron A. diluta subsp. chrysantha como notoespecie bajo 
A. $x$ chrysantha (Ehr. Bayer) J. M. Watson \& A. R. Flores, e indican los posibles padres de ella como A. kingii Phil. y $A$. diluta. No concordamos con esta propuesta, puesto que $A$. kingii se separa hoy por tener los tépalos amarillos, pero sin dibujos, o blanquecinos, pero con una franja amarilla (mapa, Fig. 3).

En la misma publicación, estos autores crean la nueva especie A. traudliae J. M. Watson \& A.R. Flores (2015), y la diferencian de $A$. $x$ chrysantha porque las rayitas cortas atropurpúreas están presentes en toda la superficie interna de los tépalos internos superiores y por el margen aserrado de los tépalos externos.

Hemos estudiado las plantas del lugar Mantos de Hornillo (localidad Tipo para A. traudliae) y los tépalos presentan solo rayitas, como las ilustraciones en el libro de Hoffmann et al. (2015) (Fig. 1F), aunque también los hay de tépalos con rayitas unidas formando una mancha. Igualmente, la variación del colorido es grande porque hay ejemplares con flores blanquecinas. Además se ha observado que el largo de los mucrones (otro carácter considerado por los autores de la especie) es muy variable, desde cortos y gruesos hasta alargados y angostos; por otra parte, el margen aserrado o no, es también muy variable. Consideramos entonces que A. traudliae es un sinónimo de $A$. diluta subsp. chrysantha (Fig. 1).

Como parte de nuestro análisis estudiamos además los ejemplares Tipo de A. citrina Phil. en SGO (descritos para Concumén en la región de Valparaíso) (Philippi 18641865) y los de la misma especie redescubiertos por M. T. Eyzaguirre (2008) en Pedernales (mapa, Fig. 3). Watson y Flores (Hoffmann et al. 2015) la comparan y diferencian de A. traudliae por sus láminas no crespas. En los ejemplares estudiados por nosotros de A. citrina, las láminas de tallos estériles son retorcidas y las de tallos floríferos no lo son o presentan una sola torsión, con los bordes lisos o algo ondulados. R. A. Philippi (1864-1865), en la descripción original de la especie, dice: "hojas de tallos estériles crespas, hojas caulinares no resupinadas". Se descarta entonces A. citrina por los detalles de sus hojas y porque generalmente presenta todos sus tépalos con rayitas atropurpúreas y sus rayos floríferos son minutamente papilosos, no glabros como en $A$. diluta.

Baeza et al. (2010) estudiaron, por otra parte, el cariotipo de A. diluta subsp. chrysantha en localidades de la región de Valparaíso, con flores de tonalidad rosada, y concluyeron después en Baeza et al. (2016) que correspondían a A. diluta subsp. diluta. Asimismo, estos autores (2016) estudiaron citotaxonómicamente esta variabilidad, tomando muestras en la región de Coquimbo, desde la provincia de Elqui (Guanaqueros) hasta la de Limarí (PN Fray Jorge), las de la región de Valparaíso antes nombradas (provincia de Valparaíso: Jardín Botánico Nacional y Punta Curaumilla) y en Maule (provincia de Talca, Río Claro). Las conclusiones de dicho trabajo, de acuerdo con la longitud de los cromosomas, son que las especies de la región de Coquimbo corresponden a la subsp. chrysantha y que las de Valparaíso al sur corresponden a la subsp. diluta. Según nuestras observaciones los ejemplares de Fray Jorge corresponden a la subsp. diluta (Fig. 2C) y además hay que considerar que los autores no tomaron muestras al norte de la región de Coquimbo.

Bayer (1987) pudo observar poco material de A. diluta subsp. chrysantha (Guanaqueros, Zorrilla, PN Fray Jorge y Ilano de Higuera); cercanos a esta última localidad (Ilanos El Tofo) hemos fotografiado y recolectado bastante material, y de Fray Jorge tenemos fotografías actuales y por ello consideramos que las especies de ambas localidades corresponden a A. diluta subsp. diluta (Figs. 2B y $2 \mathrm{C}$ )

Asimismo, observamos material procedente del camping Las Mostazas, cercano a Guanaqueros, y las flores tienen los 3 tépalos internos amarillos, pero los externos tienden al rosado. Dada la variabilidad de la especie y su cercanía con la localidad Tipo, consideramos que corresponde a A. diluta subsp. chrysantha (Fig. 1B).

Analizando las publicaciones mencionadas y los ejemplares, tanto Tipos de SGO y en parte de CONC, y gracias a las nuevas recolecciones y fotografías http:// www.fundacionphilippi.cl/search/node/alstroemeria; http:// floradechile.cl/, entendemos que solo las flores con tépalos externos amarillos, blanquecinos o algo rosados, pero los internos amarillos y generalmente con rayitas atropurpúreas dispersas corresponden a $A$. diluta subsp. chrysantha (se excluyen los ejemplares albinos, que solo tienen un leve color amarillo en los tépalos internos superiores) (Fig. 1) y que las de todos los tépalos rosados, leves o intensos hasta blanquecinos, y los internos superiores con rayitas atropurpúreas que tienden a formar una mancha, $y$ a veces con franja amarilla, corresponden a A. diluta subsp. diluta (Fig. 2) 

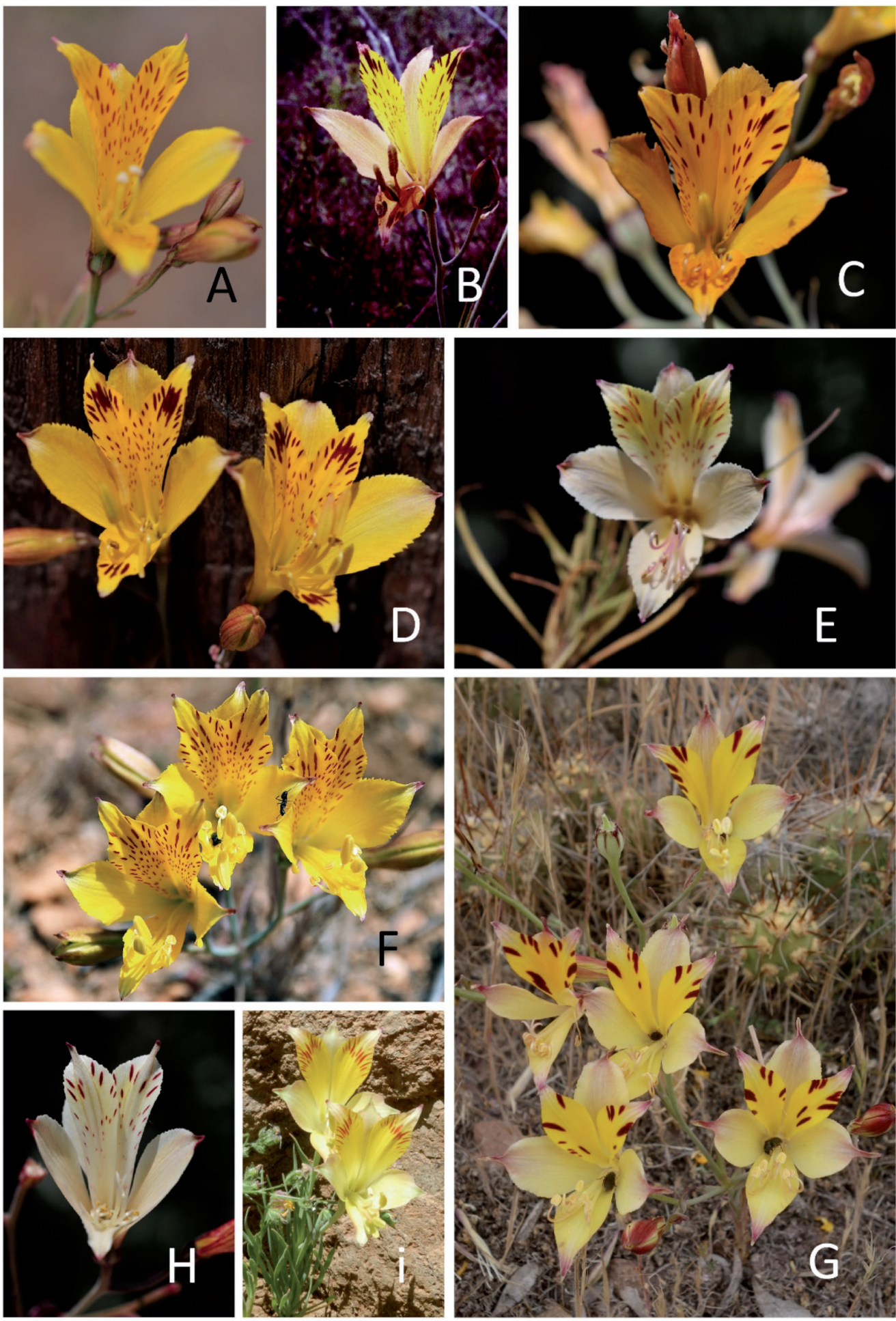

Figura 1. Alstroemeria diluta var. chrysantha. (A) Morrillos, foto S. Moreira. (B) Las Mostazas, foto S. Moreira. (C y D) Mantos de Hornillo, foto S. Moreira. (E) Mantos de Hornillo, foto S. Moreira. (F) Mantos de Hornillo, foto enviada por A.R. Flores para A. traudliae. (G) San Marcos, Ovalle, foto A. de Trenqualye. (H) Mantos de Hornillo, foto S. Moreira. (I) Quebrada La Coipa, Guatulame, foto A. de Trenqualye. / Alstroemeria diluta var. chrysantha. (A) Morrillos, photo S. Moreira. (B) Las Mostazas, photo S. Moreira. (C y D) Mantos de Hornillo, photo S. Moreira. (E) Mantos de Hornillo, photo S. Moreira. (F) Mantos de Hornillo, photo sent by A.R.Flores for A. traudliae. (G) San Marcos, Ovalle, photo A. de Trenqualye. (H) Mantos de Hornillo, photo S. Moreira. (I) Quebrada La Coipa, Guatulame, photo A. de Trenqualye. 

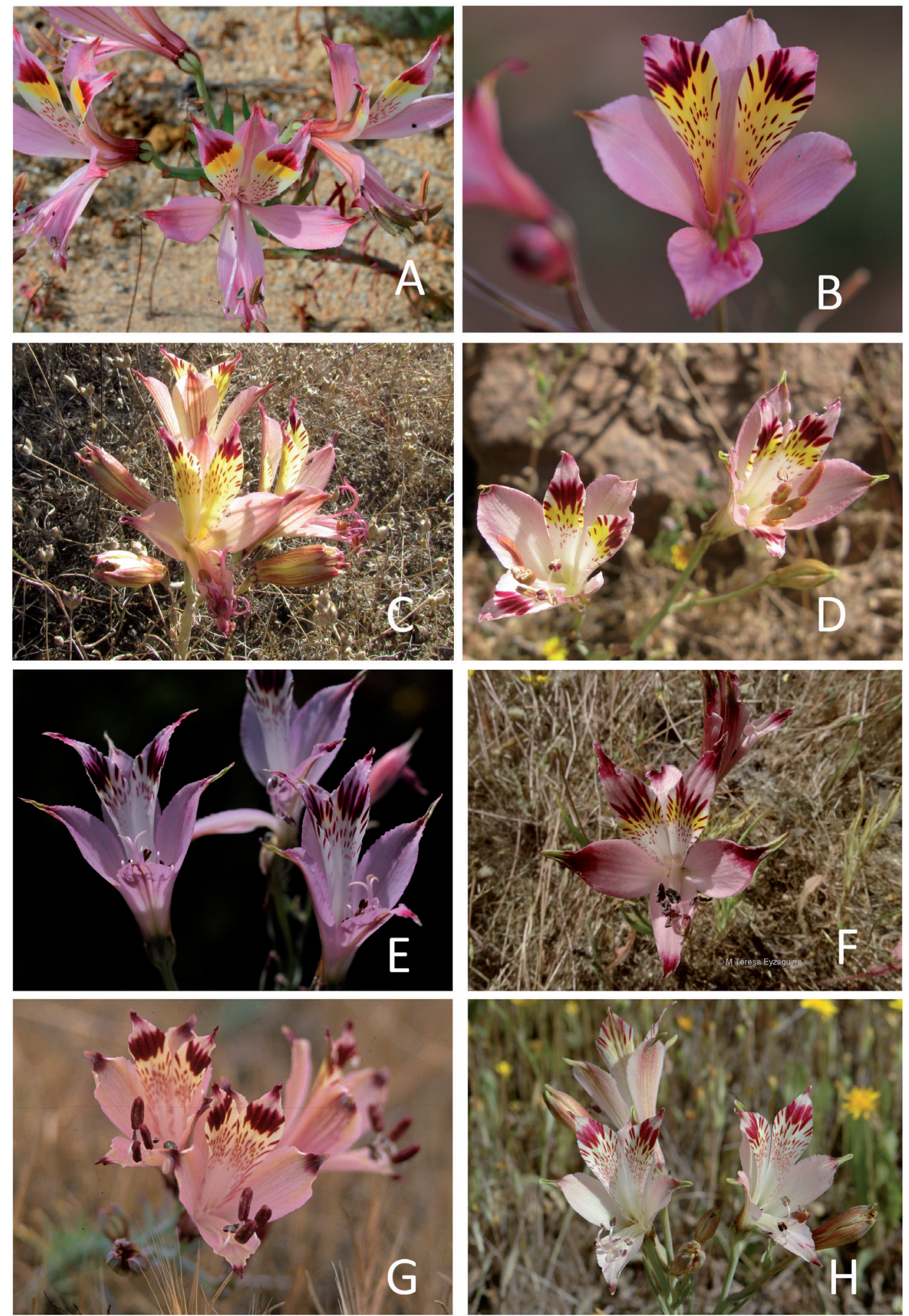

Figura 2. Alstroemeria diluta var. diluta. (A) P.N. Llanos de Challe, costa, foto A. Moreira. (B) Llanos El Tofo, foto S. Moreira. (C) Fray Jorge, foto S. Teillier. (D) Tilama, foto A. Moreira. (E) Sur Cuesta El Melón, foto S. Moreira. (F) Nogales, foto M.T. Eyzaguirre. (G) Punta Curaumilla, foto A. Moreira. (H) Lolol, foto M.T. Eyzaguirre. / Alstroemeria diluta var. diluta. (A) P.N. Llanos de Challe, coast, photo A. Moreira. (B) Llanos El Tofo, photo S. Moreira. (C) Fray Jorge, photo S. Teillier. (D) Tilama, photo A. Moreira. (E) Sur cuesta El Melón, photo S. Moreira. (F) Nogales, photo M.T. Eyzaguirre. (G) Punta Curaumilla, photo A. Moreira. (H) Lolol, photo M.T. Eyzaguirre. 


\section{CONCLUSIÓN}

Dado que este error de interpretación modificará la identidad de los ejemplares analizados, se sugieren cambios en la distribución conocida de A. diluta. La subsp. diluta amplía su distribución hacia el norte y queda entonces de $\mathrm{S}$ a $\mathrm{N}$ entre las regiones del Maule y Atacama, ya sea en la costa o al interior, tanto en terrenos arenosos como pedregosos (Fig. 3). Por otra parte, la subsp. chrysantha se distribuye solo en la región de Coquimbo en la costa, y también al interior, en las provincias de Limarí y Choapa, tanto en terrenos arenosos como pedregosos (Fig. 3).

Tomando en cuenta toda esta variabilidad en el colorido de los tépalos y ya que la subsp. chrysantha está inserta

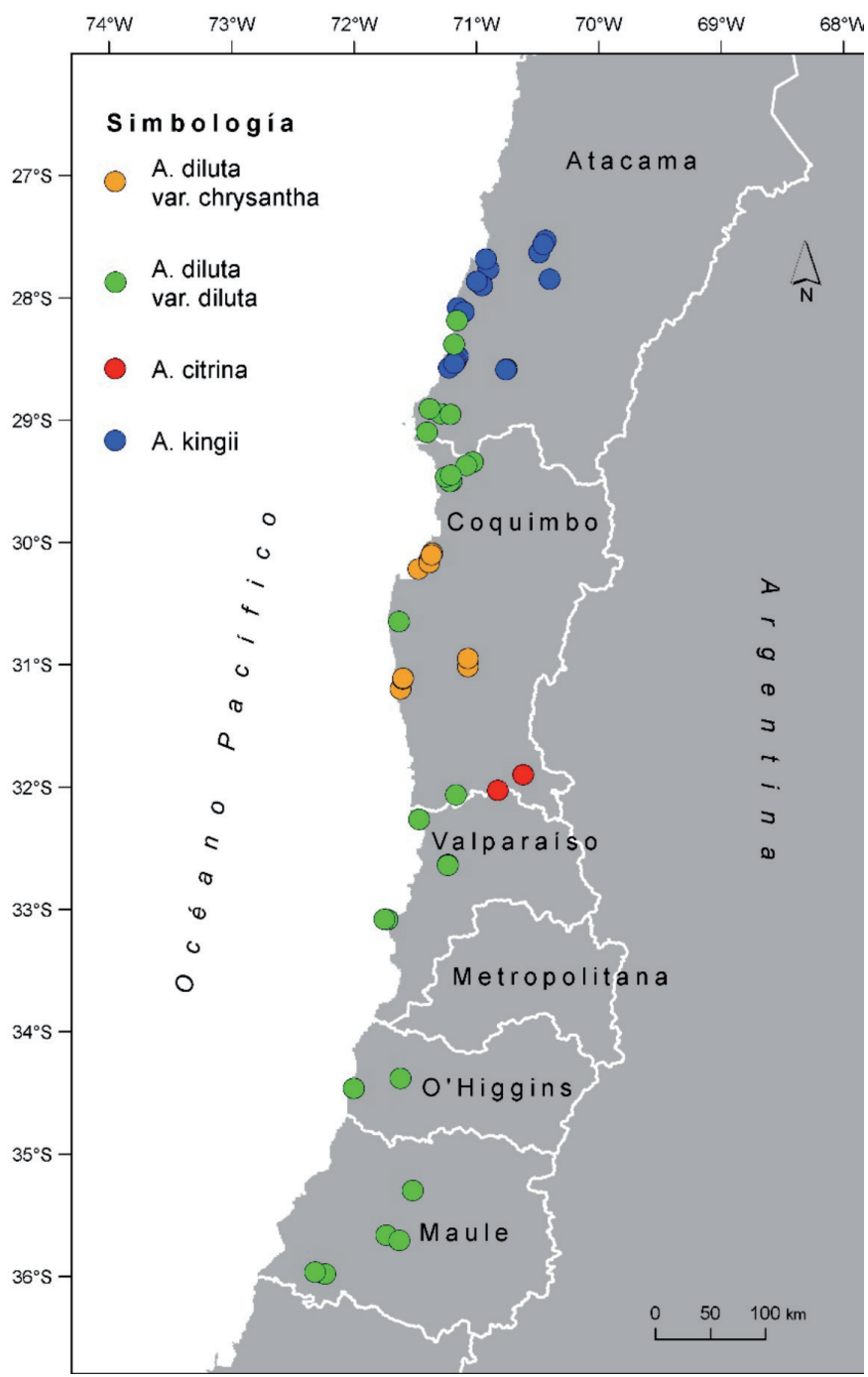

Figura 3. Mapa de las especies estudiadas. Confeccionado por Vanezza Morales. / Map of the studied species. Drawn by Vanezza Morales. dentro de la distribución general de A. diluta subsp. diluta, estimamos que deben considerarse como variedades y no como subespecies (pues en ese caso deberían separarse claramente de área), por lo cual cambian de estatus y quedan como sigue: A. diluta var. diluta y A. diluta var. chrysantha (Ehr. Bayer) Muñoz-Schick \& Moreira-Muñoz.

\section{MATERIAL REPRESENTATIVO ESTUDIADO}

\section{Alstroemeria citrina}

Tipo: provincia de Aconcagua, Concumén, Landbeck s/n, enero 1863 (SGO 47083, 47084)

Otros: provincia de Choapa, Pedernales, 1720 msnm, M. T. Eyzaguirre, 17-11-2007 (SGO 156324, 156325)

\section{Alstroemeria diluta var. chrysantha}

Tipo: región de Coquimbo, Panamericana N, Km 435, ca. Guanaqueros, Grau 2543, 27-11-1980 (imágenes de alta resolución JSTOR) (M 0219212)

Otros: región de Coquimbo: Ruta 5, $1 \mathrm{~km} \mathrm{~S}$ entrada a Las Tacas, Km 444 en Sociedad Balneario Morrillos, $\mathrm{N}$ de Lagunillas, $30^{\circ} 6^{\prime} 10^{\prime \prime} \mathrm{S}-71^{\circ} 21^{\prime} 48,3^{\prime \prime} \mathrm{W}, 38$ msnm, Muñoz M. 5546, 2-11-2015 (SGO); Coquimbo, Camping Las Mostazas, $30^{\circ} 11^{\prime}$ S $-71^{\circ} 22^{\prime}$ W, Muñoz M. y Meza I. 2210, 25-10-1987 (SGO 110622); Ruta 5, Km 320, N Mantos de Hornillo, bajada

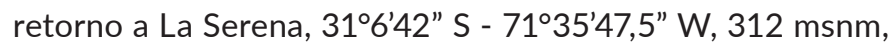
Muñoz M. 5547, 2-11-2015 (SGO); parada buses retorno a

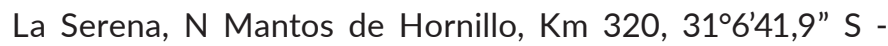
713'47,7" W, 316 msnm, Muñoz M. 5569, 22-11-2015 (SGO); Combarbalá, $12 \mathrm{~km}$ al $\mathrm{N}$ de camino a Chañaral Alto, antes de Embalse Cogotí, $31^{\circ} 0^{\prime} \mathrm{S}-71^{\circ} 0^{\prime} \mathrm{W}, 800 \mathrm{msnm}$, Muñoz M. 2562, 21-09-1991 (SGO 145252); ídem, Moreira I. $\mathrm{s} / \mathrm{n}, 21-09-1991$ (SGO 123029); $1 \mathrm{~km}$ al sur de San Marcos, $30^{\circ} 56^{\prime} \mathrm{S}-71^{\circ} 05^{\prime} \mathrm{W}$, Trenqualye A. s/n, 1-10-2017 (SGO)

\section{Alstroemeria diluta var. diluta}

Tipo: región del Maule, Talca, Panamericana S, Km 229, Grau 2394, 17-11-1980 (imágenes de alta resolución JSTOR) (M 0219211)

Otros: región de Atacama: PN Llanos de Challe, costa, 2811'9,6" S - 71'9'14,5” W, 48 msnm, Moreira A. 2803, 4-102017 (SGO); $12 \mathrm{~km}$ pasado cruce a Sarco desde Carrizalillo, $28^{\circ} 56^{\prime} 55,5^{\prime \prime} \mathrm{S}-7^{\circ} 17^{\prime} 7,9^{\prime \prime} \mathrm{W}, 309$ msnm, Muñoz M. 5517, 29-10-2015; desde cruce Sarco a Ruta 5, 2857'17,6" S 71'12'31,9" W, 388 msnm, Muñoz M. 5518, 29-10-2015; Km 8,5 después de cruce Carrizalillo-Sarco hacia Parque Eólico

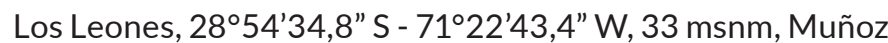
M. 5665, 3-10-2017; región de Coquimbo: primer km camino a mineral El Tofo, $29^{\circ} 26^{\prime}$ S - $71^{\circ} 18^{\prime}$ W, Muñoz et al. 2659, 27 - 
10-1991 (SGO 123804); $21 \mathrm{~km} \mathrm{~N} \mathrm{del} \mathrm{cruce} \mathrm{La} \mathrm{Higuera,} 2 \mathrm{~km}$ $\mathrm{N}$ del trapiche, $29^{\circ} 22^{\prime} 18^{\prime \prime} \mathrm{S}-7^{\circ} 4^{\prime} 45^{\prime \prime} \mathrm{W}, 357$ msnm, Muñoz M. 4252, 28-10-2002 (SGO 149256); La Serena: PN Fray Jorge, 30³8'58,9" S - 71'37'42,2" W, 298 msnm, Baxter P. et al. 1796, 2-12-2004 (SGO 162687); puente El Mantillo,

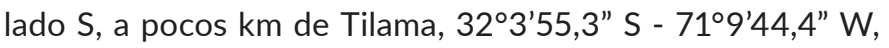
520 msnm, Moreira A. 2542, 22-11-2015 (SGO); región de Valparaíso: Los Molles, $32^{\circ} 13^{\prime} \mathrm{S}-7^{\circ} 30^{\prime} \mathrm{W}$, Moreira A. 2548, 11-12-2015 (SGO); Punta Curaumilla al norte del faro, 335'13" S - 7143'31" W, Moreira A. 817, 3-12-2002 (SGO 150070); región Metropolitana: S cuesta El Melón, Km 127, $32^{\circ} 38^{\prime} 28,7^{\prime \prime}$ S - 71¹3'53,2" W, 260 msnm, Muñoz M. 5584, 8-12-2015 (SGO); región de O'Higgins: Marchihue, 34²3' S - 71³8' W, Frindt G. s/n, 26-11-1999 (SGO 145344); región del Maule: Subestación Experimental Cauquenes INIA,

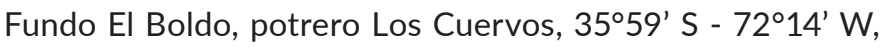
177 msnm, Muñoz M. 1475, 7-11-1979 (SGO 108327)

\section{Alstroemeria traudliae}

Tipo: región de Coquimbo, Ruta $5 \mathrm{~N}, 4 \mathrm{~km} \mathrm{~N}$ Mantos de Hornillo, Flores y Watson 9524, 8-11-2000 (SGO 169846)

\section{AGRADECIMIENTOS}

Dedicamos esta publicación a nuestro recordado amigo Alain de Trenqualye, q.e.p.d., quien nos colaboró con observaciones y fotografías. A Sergio Moreira y María Teresa Eyzaguirre por sus excelentes fotografías. A Sebastián Teillier, por fotografía de la especie de Fray Jorge, y a Ana R. Flores, por la foto de A. traudliae. A Vanezza Morales, por el mapa de distribución. Al revisor o revisora anónima por sus acertadas sugerencias. Al Proyecto Fondecyt 1180211.

\section{REFERENCIAS}

Baeza, C.M., Ruiz E., Novoa, P. 2010. Karyotype of Alstroemeria diluta Ehr. Bayer subsp. chrysantha (Alstroemeriaceae). Chilean Journal of Agricultural Research 70(4): 667-669.

Baeza, C.M., Finot, V.L., Ruiz, E., Carrasco, P., Novoa, P., Rosas, M. 2016. Análisis citotaxonómico del complejo Alstroemeria diluta E. Bayer (Alstroemeriaceae) de Chile. Gayana Botánica 73(2): 220-225.

Bayer, E. 1987. Die Gattung Alstroemeria in Chile. Mitteilungen der Botanischen Staatssammlung München 24: 1-362.

Eyzaguirre, M.T. 2008. Validación de Alstroemeria citrina Phil. (Alstroemeriaceae). Gayana Botánica 65(2): 241-244.

Finot, V., Baeza, C., Muñoz-Schick, M., Ruiz, E., Espejo, J., Alarcón, D., Carrasco, P., Novoa, P., Eyzaguirre, M.T. 2018. Guía de campo, Alstroemerias Chilenas. CORMA. 295 pp.

Hoffmann, A., Watson, J., Flores, A.R. 2015. Flora silvestre de Chile. Cuando el desierto florece. Vol. 1, Fundación C. Gay. 263 pp.

Muñoz-Schick, M., Moreira-Muñoz, A. 2003. Alstroemerias de Chile. Diversidad, distribución y conservación. Taller La Era - Consejo Nacional del Libro y la Lectura. Santiago. $140 \mathrm{pp}$.

Muñoz-Schick, M., Eyzaguirre, M.T., Moreira-Muñoz, A. 2019. Redescubrimiento de una especie y su nueva clasificación como subespecie de Alstroemeria garaventae (Alstroemeriaceae) de Chile. Gayana Botánica 76(2): 247-252.

Philippi, R.A. 1864-1865. Plantarum novarum chilensium. Linnaea 33: 1-308, Taf. I-III. (Alstroemeria citrina: 264-265).

Received: 13.04.2019

Accepted: 30.07.2020 